\title{
Evaluation of Blue Light Hazard of Luminaires by Spline Function Fitting
}

\author{
Shikang Zhou", Jin Du, Shan Li, Xing Wen, Xiaoliang He
}

Shanghai Sansi Electronic Engineering Technology Co. Ltd, Shanghai, China

Email address:

2447441104@qq.com (Shikang Zhou)

${ }^{*}$ Corresponding author

To cite this article:

Shikang Zhou, Jin Du, Shan Li, Xing Wen, Xiaoliang He. Evaluation of Blue Light Hazard of Luminaires by Spline Function Fitting. Optics. Vol. 9, No. 1, 2020, pp. 8-12. doi: 10.11648/j.optics.20200901.12

Received: October 25, 2020; Accepted: November 30, 2020; Published: December 4, 2020

\begin{abstract}
White LEDs are usually excited by blue light. The wide spread use of LED table lamps, made people pay more and more attention to blue light hazard of luminaires. In fact, blue light harms human retina only if irradiation exceeds a certain limit. The problem is therefore to precisely characterize the blue light hazard of a luminaire. In general, blue light hazard is amenable to measurements, but the corresponding detection schemes are complex and expensive. In order to overcome this issue, we put forward a mathematical fitting method to evaluate blue light hazard. It consists of fitting the spectrum of a lamp using spline functions to obtain the lamp brightness. At the same time, spline function approach is also used to obtain the weight function expression of blue light hazard starting from data listed in IEC/EN62471. Finally, a blue light hazard function with wavelength as an independent variable is introduced. The damage due to blue light exposure may be then evaluated by integrating this function. In this way, as long as the absolute light power spectrum of a luminaire is known, quantification of blue light hazard may be directly obtained without measurements. Our method provides a convenient technique to evaluate and analyze the safety level of lamps before production. In order to assess the reliability of our method, we use it to evaluate the blue light hazard of two lamps, which belong to IEC/EN62471 safety level RG0 and RG1 respectively. Results are in good agreement with those measured by authoritative departments. In this way, evaluation of blue light hazard may be obtained by the knowledge of absolute physical properties of the source, a feature which definitely provides an optional approach to assess the photobiological safety of LED lamps.
\end{abstract}

Keywords: Blue Light Hazard, Photobiological Safety, LED Lamp, Spline Function Fitting

\section{Introduction}

Safety is a crucial aspect of the of LED lamps. Correspondingly, the evaluation of their potential hazard is a relevant field of research. In particular, the widespread application of LED lights made the evaluation of blue light hazard very relevant. In fact, white LEDs are excited by blue light, and concerns have arisen about the potential harm of blue light to human eyes. However, evaluation of blue light hazard may be challenging, since it requires the use of many separated data points. Calculations may be carried out on a computer, but in order to improve accuracy, interpolation must be used, which greatly increases the complexity.

In this paper, the existing calculation methods are improved, in order to simplify the calculation and improve the accuracy. Spline functions are used to fit data points and obtain functional expressions. The quantitative blue light hazard value may be then obtained directly without any measurement. Overall, our results provide an additional simple tool to address photobiological safety assessment of lamps and lanterns.

\section{Blue Light Hazard}

Blue light irradiation is known to cause damages to retinal cells, leading to vision weakening or even loss. Short-wave blue light, with wavelength in the range $400-480 \mathrm{~nm}$, is the most harmful to the retina. In fact, blue light irradiation of the retina produces free radicals, which can lead to the death of retinal pigment epithelial cells. This will result in a lack of nutrients in photosensitive cells, causing visual impairment, usually irreversible. 
Because white LEDs are excited by blue light, the blue light hazard of LED is a problem that needs to be addressed and analyzed. Among all kinds of luminaires, LED desk lamps are those closest to human eyes, and thus deserve special attention [1].

The key to whether blue light is harmful depends on the "quantity" of light impinging on the eye. In fact, blue light itself is not necessarily harmful to the eyes, but too much blue light is indeed harmful. If the dose is large enough, any light is going to cause visual fatigue, or even causes cataract and macular lesions.

The basis to determine how much blue light radiation is harmful to people is the standard IEC/EN62471 developed by the European Union and the International Electrotechnical Commission.

\section{Risk Groups}

The current effective international standards for blue light research are IEC/EN 624712008 [2], IEC60598.1 2014 [3] and IEC TR 62778: 2014 [4]. GB 7000.1. According to the standard [2], the blue light retinal hazards of continuous light source lamps are divided into four groups according to the degree of risk:

RG0: No hazard category (radiance $\leq 100 \mathrm{Wm}^{-2} \mathrm{Sr}^{-1}$ ), The lamp does not cause any photo biological hazards if the radiance is below this limit.

RG1: Low risk category (radiance $\leq 10^{4} \mathrm{Wm}^{-2} \mathrm{Sr}^{-1}$ ). The lamp does not cause harm under normal exposure conditions. RG2: Medium risk category (radiance $\leqslant 4 \times$ $\left.10^{6} \mathrm{Wm}^{-2} \mathrm{Sr}^{-1}\right)$. The lamp does not produce harm, thanks to the normal aversion response reflexes to bright light and heat (this is the highest classification permitted for consumers)
RG3: High risk category (radiance $>4 \times 10^{6} \mathrm{Wm}^{-2} \mathrm{Sr}^{-1}$ ). The lamp may easily cause harm in a short time.

Whether a lamp is going to produce any harm depends on which group it conforms to. Any claim related to "no blue light" LED is unscientific and unnecessary, despite the attitude of some manufacturers. As long as a lamp is classified as RG0, we may safely conclude that the lamp is harmless.

Due to its relevance, the photobiological safety of luminaires has been the subject of several works [6-8], and many are dedicated to the evaluation of blue light hazard [9$11]$.

\section{Calculation Basis}

The measurement of blue light hazard may be complex, and the involved instruments are generally very expensive. If one only needs to know which safety group the luminaire belongs to, the method indicated in IEC62778 may be employed. Zhu Chunhong [12] and others have analyzed and the blue light hazard of many LED lights based on IEC62778.

However, if one want to know the retinal blue light hazard exposure limit $L_{B}$, the following formulas from IEC62461 [2] should be used.

$$
\begin{gathered}
L_{B}=\sum_{300}^{700} \sum_{\lambda}(\lambda, t) B(\lambda) \Delta t \Delta \lambda \leq 10^{6}\left(\mathrm{Jm}^{-2} \mathrm{Sr}^{-2}\right), t \leq 10^{4} s \\
L_{B}=\sum_{300}^{700} L_{\lambda} B(\lambda) \Delta \lambda \leq 100\left(\mathrm{Wm}^{-2} \mathrm{Sr}^{-2}\right), t>10^{4} s
\end{gathered}
$$

where $L_{\lambda}(\lambda, t)$ is the spectral radiance $\left(\mathrm{W} \mathrm{m} \mathrm{Sr}^{-1} \mathrm{~nm}^{-1}\right)$, $B(\lambda)$ is the hazard weight of blue light, which is listed in Table $1, \Delta \lambda$ is the bandwidth (nm), and $\mathrm{t}$ is the exposure time (seconds).

\begin{tabular}{|c|c|c|c|}
\hline Wavelength/nm & Blue Light Hazard Weight B & Wavelength/nm & Blue Light Hazard Weight B \\
\hline 300 & 0.01 & 405 & 0.20 \\
\hline 305 & 0.01 & 410 & 0.40 \\
\hline 310 & 0.01 & 415 & 0.80 \\
\hline 315 & 0.01 & 420 & 0.90 \\
\hline 320 & 0.01 & 425 & 0.95 \\
\hline 325 & 0.01 & 430 & 0.98 \\
\hline 330 & 0.01 & 435 & 1.00 \\
\hline 340 & 0.01 & 445 & 0.97 \\
\hline 345 & 0.01 & 450 & 0.94 \\
\hline 350 & 0.01 & 455 & 0.90 \\
\hline 355 & 0.01 & 460 & 0.80 \\
\hline 360 & 0.01 & 465 & 0.70 \\
\hline 365 & 0.01 & 470 & 0.62 \\
\hline 370 & 0.01 & 475 & 0.55 \\
\hline 380 & 0.01 & 485 & 0.40 \\
\hline 385 & 0.013 & 490 & 0.22 \\
\hline 390 & 0.025 & 495 & 0.16 \\
\hline 395 & 0.05 & $500 \sim 600$ & $10^{[(450-\lambda) / 50]}$ \\
\hline 400 & 0.10 & $600-700$ & 0.001 \\
\hline
\end{tabular}

Table 1. Blue light hazard weight B.

For lamps with continuous wavelength, the time $t$ can be removed, the exposure limit $L_{B}$ in formula (1) may be simplified to the following summation:

$$
L_{B}=\sum_{300}^{700} L(\lambda) B(\lambda) \Delta \lambda
$$


However, in order to improve the accuracy, it is necessary to get as much spectral distribution data $L_{\lambda}(\lambda)$ as possible. For example, for $\Delta \lambda=1 \mathrm{~nm}, 500$ points are needed in the visible light range $300-800 \mathrm{~nm}$. One also needs to know the value of blue light hazard weights $B(\lambda)$ for 500 wavelengths, which requires additional interpolation. This kind of calculations may become challenging and, more importantly, not convenient for fast or multi-task computing.

In this paper, a new convenient method to calculate the hazard of blue light is proposed. Two spline functions $L(\lambda)$ and $B(\lambda)$ are used, leading to functional expressions that facilitate mathematical calculations. They are still fitted from data points, but do not need 500 points, only about $100 \mathrm{~L}$ points and $60 \mathrm{~B}$ points.

For the sake of convenience, let us introduce the following blue light hazard function $H(\lambda)$

$$
H(\lambda)=L(\lambda) B(\lambda)
$$

In this way, the sum in Eq. (2) becomes an integral of $H(\lambda)$ in (3). This makes it easier to calculate the blue light hazard as

$$
L_{B}=\int_{\lambda 1}^{\lambda 2} L(\lambda) B(\lambda) d \lambda=\int_{\lambda 1}^{\lambda 2} H(\lambda) d \lambda
$$

\section{Spline Function}

Splines are special functions with a wide range of uses [13]. They are piecewise low-order polynomial functions, and their nodes are the data points. The "order" of the polynomial can be selected according to the requirements of calculation speed and accuracy. Splines involve low order polynomials to obtain smooth single-valued functional expressions, which makes it easier to manipulate the involved quantities. Splines provide accurate and flexible interpolation, with several advantages compared to many other commonly used interpolation methods. Splines are suitable for most kinds of regular data. Their disadvantages being that the expression may be very long, and difficult to obtain by hand, though it may be easily calculated by mature mathematical software.

The method used in this work relies on spline function fittings of spectral brightness data and blue light hazard weight table. After obtaining the corresponding mathematical expression, the tedious summation turns into simple integration, which is very easy on the mathematical platform. Another important advantage is that we don't need so many data points to get more accurate results. For example, the calculation accuracy based on 100 measurement points is much higher than that of the summation method with 500 points.

The spline functions used in calculations are third-order polynomials by default, and the corresponding errors are relatively small. The standard errors of the two examples are estimated to be about $0.004 \%$ and $0.001 \%$, respectively.

\section{Calculation Method}

a. The measured data points of absolute spectral radiance are fitted by a spline function to get the piecewise function expression $\mathrm{L}(\lambda)$.

b. The blue light hazard weight function $B(\lambda)$ is obtained by fitting the blue light hazard weight data in Table 1 .

c. The product of the two functions is the blue light hazard function $H(\lambda)$.

d. The blue light hazard exposure amplitude $L_{B}$ is then obtained by integrating $H(\lambda)$ using formula (3).

The above steps can be easily implemented in automatic calculations.

If no measurement data are available, but only spectral curve graph, the spectral curve can be converted into two column data matrix by data curve analysis software. There are many kinds of software for data curve analysis, which are not analyzed here.

\section{Two Examples}

Sample 1 is C22RL-TD-02, the intelligent voice eye lamp of Shanghai Sansi Company. The blue light retina hazard of this luminaire is RG0. Sample 2 is Sansi C22RL-TD-07 lamp, belonging to RG1 class. These are the results of authoritative measurement. According to IEC/EN 62471 2008 [2], these light sources are measured at a field-of-view angle of $11 \mathrm{mrad}$ at a position of $200 \mathrm{~mm}$.

Our results may be presented in three equivalent ways, i.e. showing data, functions, or graphics. These three ways may be easily converted into each other. In order to show results visually, we report plots of blue light retina hazard evaluation.

\subsection{Spectral Power Function of Lamps}

The power spectrum fitting is performed using $n=121$ data pairs $(\mathrm{X}, \mathrm{Y})$, where $\mathrm{X}$ represents the wavelength $(\mathrm{nm})$ and $\mathrm{Y}$ represents the power $(\mathrm{W})$. In the mathematical software Maple, the command for spline function $L(\lambda)$ to fit these data points is:

$$
L(\lambda)=\text { CurveFitting }[\text { Spline }](X, Y, \lambda)
$$

Only the command line of the spline function is listed here, whereas the specific expression is not listed for brevity. $\mathrm{L}(\lambda)$ may be easily displayed graphically, as shown in Figure 1. This provides the spectral radiance function of the luminaire. 


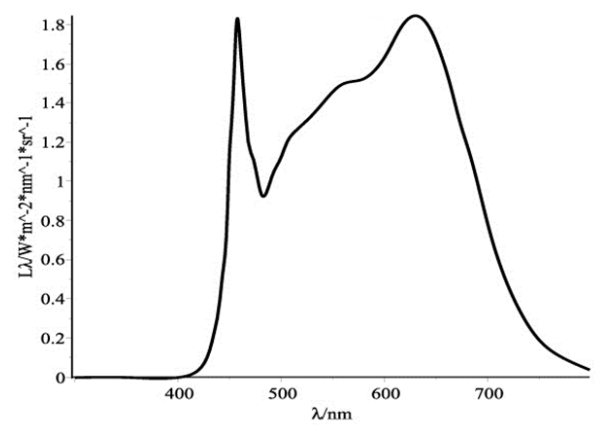

Sample1

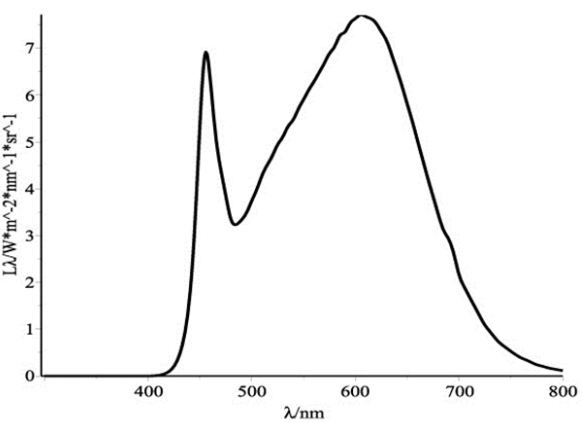

Sample 2

Figure 1. Spectral radiance function $L(\lambda)$.

\subsection{Blue Light Hazard Weight}

The wavelength and blue light hazard weights in Table 1 are 61 data $\mathrm{Xb}$ and $\mathrm{Yb}$, respectively, and the blue light hazard weight fitting function $B(\lambda)$ is:

$$
B(\lambda)=\text { CurveFitting }[\text { Spline }](X b, Y b, \lambda)
$$

The function $B(\lambda)$ is in Figure 2.

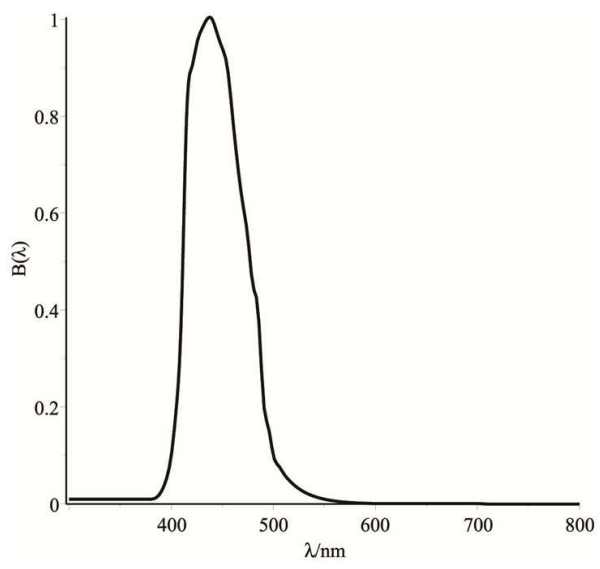

Figure 2. Blue light hazard weight function $B(\lambda)$.

\subsection{Blue Light Hazard Function}

By multiplying (5) and (6), the expression of the blue light hazard function $H(\lambda)$ is obtained.

Figure 3 shows the blue light hazard function $H(\lambda)$ of the two test lamps. It can be seen that the blue light hazard function of sample 2 is much larger than that of sample 1.

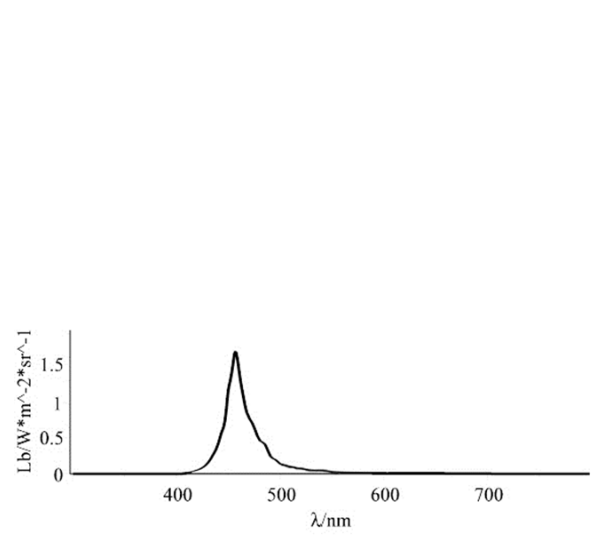

Sample 1

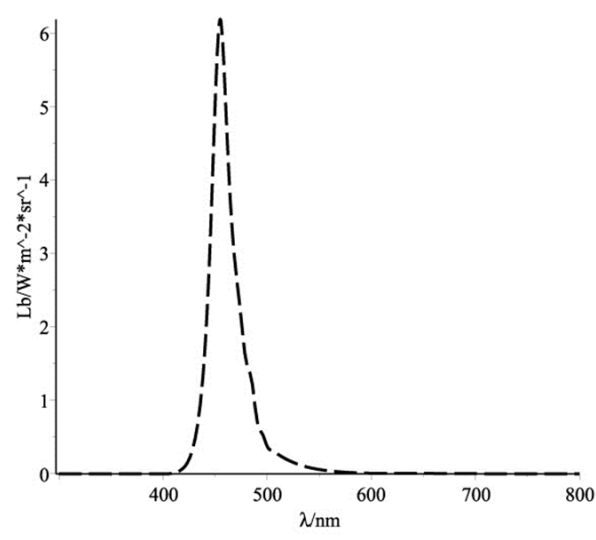

Sample 2

Figure 3. Blue light hazard function $H(\lambda)$. 


\subsection{Blue Light Weighted Irradiation Value}

We evaluate blue light hazard exposure amplitude $L_{B}$ using formula (4). The calculated blue light weighted irradiation value of sample 1 is $L_{B}=47.061\left(W^{-2} \mathrm{Sr}^{-1}\right)$, whereas for sample 2 we obtain $L_{B}=187.524\left(\mathrm{~W} \mathrm{~m}^{-2} \mathrm{Sr}^{-1}\right)$. According to the international and domestic standards, sample 1 meets the hazard level of RG0, that is, it is harmless. The hazard level of sample 2 luminaire is RG1 that is low risk category. Using standard test conditions $(200 \mathrm{~nm}$, $0.011 \mathrm{rad}$ field of view) at Detection Center of Shanghai Lighting Electrical Testing Co., Ltd., the measured blue sample weighted irradiance values of the two samples are $48.104\left(W^{-2} \mathrm{Sr}^{-1}\right) \quad$ and $\quad 187.1\left(\mathrm{~W} \mathrm{~m}^{-2} \mathrm{Sr}^{-1}\right)$ respectively. Compared with the calculated results, they are highly consistent. The difference is only $1.04 \%$ and $0.23 \%$, respectively.

\section{Conclusions}

1) By using spline function, the weight of blue light hazard and the radiant brightness of luminaires may be accurately transformed into functional expressions, here termed $\mathrm{B}(\lambda)$ and $\mathrm{L}(\lambda)$, respectively. The blue light hazard function $H(\lambda)=L(\lambda) B(\lambda)$ of a lamp has been introduced, and the blue light hazard value $L_{B}$ can be obtained by integrating $H(\lambda)$.

2) Due to the complexity of measurement, the accuracy of blue light hazard measurement depends on the accuracy of spectral radiation analyzer, retinal luminance meter, standard light source, etc. The corresponding uncertainty may be as high as $20-30 \%[14,15]$. On the contrary, because of the high fitting accuracy of spline function, and the improved accuracy of integration compared to summation, our method provide higly reliable results. Overall, our approach provides an effective way to obtain blue light hazard without measurements.

\section{References}

[1] ZHANG Yuemin QIAO Gengxin, Photobiological Safety of blue light in LED lighting [J]. China Light \& Lighting, 2013, 6: 2-3.
[2] Photobiological safety of lamps and lamp systems: IEC62471: 2006 [S]. IEC, 2006.

[3] Luminaires-Part 1: General requirements and tests: IEC 60598-1: 2014 [S]. IEC 2014.

[4] IEC TR 62778: 2014. Application of IEC 62471 for the assessment of blue light hazard to light sources and luminaires [S].

[5] GB 7000.1: 2015/IEC60598-1: 2014. [5] General requirements and tests for luminaires [S].

[6] Chen Huiting Cai Zhe $\mathrm{Wu}$ Xiaochen Peng Zhenjian Huang Jun, Photobiological Safety Test and analysis of high power LED street lamp [J], China Illuminating Engineering Journal, 2011, 26 (6), 88-96.

[7] KONG Qiangqiang; SONG Peng; WANG Meixia; TAN Chen, Photobiological Safety Test and analysis of LED lamps [J], 2014, $10(38-42)$.

[8] Yu Jiandong, Testing and evaluation of Photobiological Safety [D], Master's thesis of Zhejiang University. 2006, 5.

[9] Jung, Myoung \& Yang, Seok-Jun \& Yuk, Ju \& Oh, SangYoung \& Kim, Chang-Jin \& Lyu, Jungmook \& Choi, Eun. (2015). Evaluation of Blue Light Hazards in LED Lightings [J]. Journal of Korean Ophthalmic Optics Society. 20. 293300 .

[10] Yang, C.-P \& Fang, W.-Q \& Liu, M.-B \& Li, C. \& Zhang, M.L \& Han, X. \& Liu, Y.-F \& Dai, W.-L. (2018). A Visualization Evaluation Method for Blue Light Hazard and Circadian Effect of Light Source [J]. Spectroscopy and Spectral Analysis. 38. 3476-3482.

[11] CIE 127-2007. Measurements of LEDs [S].

[12] ZHU Chunhong, YU Tingyu, CHEN Wencong, MAI Yong,(2016). Application Analysis of IEC 62778 on National Compulsive Standard of Luminare [J], China Illuminating Engineering Journal, 27, 122-126.

[13] Popular science in China Science Encyclopedia entry compilation and application project group, Spline function [R], Baidu Encyclopedia (updated on January 20, 2018).

[14] SANSE Co., Ltd, Zhejiang University, photobiosafety testing system training materials [OL], 2019, 11, 19 p30.

[15] Private communication: The actual comparison of the error of blue light weighted radiation value measured by different domestic companies for the same lamp has reached more than $30 \%$ [Z], June 2020 . 